\title{
The Next Generation Mobile Wireless Cellular Networks $-4 G$ and Beyond
}

\author{
Arun Agarwal $^{1, *}$, Kabita Agarwal ${ }^{2}$ \\ ${ }^{1}$ Department of Electronics \& Communication Engineering, Institute of Technical Education and Research, Siksha ‘O’ Anusandhan \\ University, Khandagiri Square, Bhubaneswar, India \\ ${ }^{2}$ Department Electronics \& Telecommunication Engineering, CV Raman College of Engineering, Bhubaneswar, India \\ *Corresponding author: arunagrawal@soauniversity.ac.in
}

Received April 25, 2014; Revised April 29, 2014; Accepted April 29, 2014

\begin{abstract}
The demand of mobile user is ever increasing in this world of digital systems. Consumers demand more from their technology. Whether it is a television, cellular phone, Washing machine or refrigerator, the latest technology purchase must have new features. With the advent of the Internet, the most-wanted feature is better, faster access to information. The time not far away when access to all necessary information and the power of a personal computer, sits in the palm of one's hand. To support such a powerful system, we need pervasive, highspeed wireless connectivity. The drawbacks of the current mobile communication technology have led the researchers to come up with more advanced and efficient technologies. 4G mobile technology is the outcome in this direction. $4 \mathrm{G}$ is the next generation of wireless networks that will totally replace existing $3 \mathrm{G}$ networks. It is supposed to provide its customers with better speed and all IP based multimedia services. In the present scenario existing technologies are capable of performing functions like broadband data access, supporting voice traffic using voice over IP (VoIP), in mobile environment etc., but there is a great requirement of integrating all such technologies into a single combined system. 4G promises a solution to this problem by seamlessly integrating the terminals, networks and applications. This paper presents an overall study of the $4 \mathrm{G}$ systems, architecture, standard, benefits, challenges in implementation, design for the LTE and WiMAX technologies, Security issues and future scope of 4G technologies.
\end{abstract}

Keywords: CDMA, IP, LTE, multimedia services, security, TDMA, WiMAX, 3G, and 4G wireless

Cite This Article: Arun Agarwal, and Kabita Agarwal, "The Next Generation Mobile Wireless Cellular Networks - 4G and Beyond.” American Journal of Electrical and Electronic Engineering, vol. 2, no. 3 (2014): 92-97. doi: 10.12691/ajeee-2-3-6.

\section{Introduction}

The Mobile devices are getting smaller, lighter, and more powerful; they have bigger screens and longer battery life, more features and more capabilities. Bandwidth will always be the limiting factor in the development of applications and devices, be it wired, or wireless. The short fall of $3 G$ networks is clear, it's just not fast enough, offering 384kbps doesn't meet the requirements of what the end user has come to expect these days. Some people see 3G as a stop-gap, until a fully integrated IP network is created; some countries have even chosen to bypass $3 \mathrm{G}$ and head straight to $4 \mathrm{G}$, a method which has its advantages, and its disadvantages.

4G technology is actually an integrated, global network that will be able to provide a comprehensive IP solution where voice, data and streamed multimedia can be given to users on an "Anytime, Anywhere" basis [1]. The next generation of wireless technology (fourth generation or $4 \mathrm{G}$ ) is intended to support broadband performance and enable voice/video multimedia applications. The enabling technologies and standards for 4G wireless communications allow for significant increases in data rates over 2G (second generation), 3G (third generation) and 3.5G wireless technologies. At the present time, LTE (Long Term Evolution) and WiMAX (Worldwide Interoperability for Microwave Access) are the two technologies considered as candidates to achieve the 4G wireless performance objectives [2].

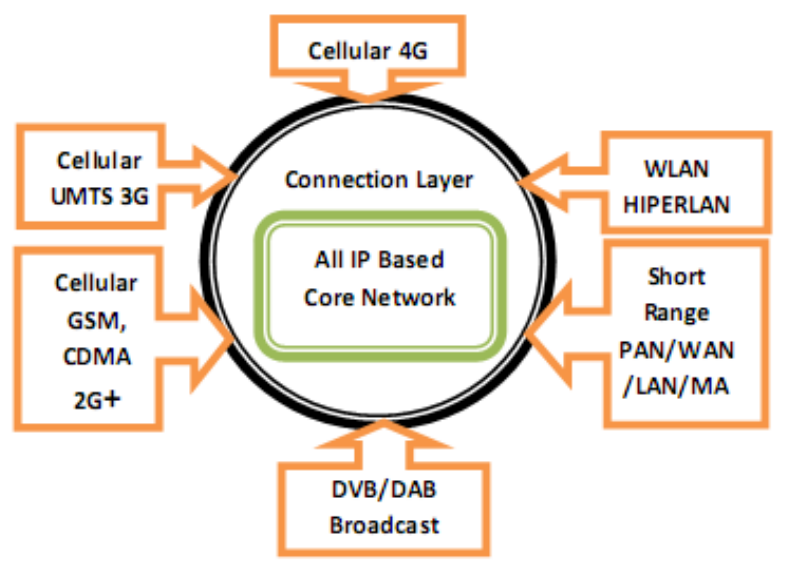

Figure 1. Seamless connections of networks [1] 
These objectives include: that $4 \mathrm{G}$ will be a fully IPbased integrated system. 4G will be capable of providing between $100 \mathrm{Mbit} / \mathrm{s}$ and $1 \mathrm{Gbit} / \mathrm{s}$ speeds both indoors and outdoors, with premium quality and high security. The term $4 \mathrm{G}$ is used broadly to include several types of broadband wireless access communication systems, not only cellular telephone Systems [1]. One of the terms used to describe $4 \mathrm{G}$ is MAGIC-Mobile multimedia, anytime anywhere, Global mobility support, integrated wireless solution, and customized personal service. As a promise for the future, 4G systems, that is, cellular broadband wireless access systems have been attracting much interest in the mobile communication arena. The 4G systems not only will support the next generation of mobile service, but also will support the fixed wireless networks [4]. Figure 1 illustrates the seamless connectivity of the different Networks to IP.

\section{Evolution of Mobile Radio}

After a brief introduction to $4 \mathrm{G}$, this section briefly outlines the evolution of mobile wireless technology from $1 \mathrm{G}$ (First Generation) to 4G.

\subsection{G}

This term refers to the first generation cellular systems that were analog telecommunications standards introduced in the $1970 \mathrm{~s}$. The voice channel typically used frequency modulation, and they used FDMA techniques. Some of the standards are NMT (Nordic Mobile Telephone), AMPS (Advanced Mobile Phone System) TACS (Total Access Communications System) and C-450. The major drawbacks were Poor voice quality, Poor battery life, large phone size, No security, frequent call drops, Limited capacity and poor handoff reliability.

Table 1. Evolution of mobile radio standards [1]

\begin{tabular}{|c|c|c|c|c|c|}
\hline Tchnology & $1 \mathrm{G}$ & $2 \mathrm{G}$ & $25 \mathrm{G}$ & $3 \mathrm{G}$ & $4 G$ \\
\hline Year & 1970 & 1980 & 1985 & 1990 & 2000 \\
\hline Standards & $\begin{array}{l}\text { AMPS, NMT, Hicap, } \\
\text { CDPD, TACS, ETACS }\end{array}$ & $\begin{array}{l}\text { GSM, i-DEN, } \\
\text { D-AMPS }\end{array}$ & EDGE, GPRS & CDMA 2000,WCDMA & $\begin{array}{c}\text { Single Standard LTE- } \\
\text { Advanced }\end{array}$ \\
\hline $\begin{array}{c}\text { Data } \\
\text { Bandwidth }\end{array}$ & $1.9 \mathrm{kbps}$ & $14.4 \mathrm{kbps}$ & 384 kbps & $2 \mathrm{Mbps}$ & $200 \mathrm{Mbps}$ \\
\hline Core Network & PSTN & PSTN & PSTN packet network & packet network & INTERNET \\
\hline Multiplexing & FDMA & CDMA TDMA & TDMA CDMA & CDMA & OFDMA \\
\hline Service & Analog Voice & Digital Voice & $\begin{array}{l}\text { Packetized Data, } \\
\text { Higher Capacity }\end{array}$ & $\begin{array}{l}\text { Higher Capacity, } \\
\text { Broadband Data }\end{array}$ & $\begin{array}{c}\text { Broadband Data With } \\
\text { High Speed }\end{array}$ \\
\hline
\end{tabular}

\section{2. $2 \mathrm{G}$}

This term refers to the second generation cellular telecommunications systems that were introduced in 1980 s. The systems were digital and were oriented to voice with only low speed data services. Systems such as GSM and US-TDMA used a mixture of TDMA and FDMA techniques. IS-95 /cdmaOne were the first CDMA system. $2 \mathrm{G}$ services are frequently referred as Personal Communications Services, or PCS, in the United States. $2 \mathrm{G}$ phones used global system for mobile communications (GSM) standard. The GSM is a circuit switched, connection oriented technology, where the end systems are dedicated for the entire call session. This causes inefficiency in usage of bandwidth and resources. The GSM-enabled systems do not support high data rates. They are unable to handle complex data such as video.

\section{3. $2.5 G$}

This term is used to describe 2G-systems that have implemented a packet switched domain in addition to the circuit switched domain. 2.5G is not an officially defined term rather it was invented for marketing purpose [1]. These systems enable high-speed data transfer over upgraded existing 2G networks. Examples: EDGE, GPRS, CDMA2000.

\subsection{G}

This refers to Third generation. The designation for systems following the 2G systems and they offer high speed data services in addition to the basic voice capability.

Typically they use CDMA techniques and include UMTS (W-CDMA), CDMA2000 1xEV-DV, CDMA2000 1xEV-DO, and TD-SCDMA. The 3G technology adds multimedia facilities to 2G phones by allowing video, audio, and graphics applications. Over $3 G$ phones, you can watch streaming video or have video telephony. 3G promises increased bandwidth, up to $384 \mathrm{kbps}$ when the device holder is walking, $128 \mathrm{kbps}$ in a car, and 2 Mbps in fixed applications. However the short comings of $3 \mathrm{G}$ networks were High bandwidth requirement, High spectrum licensing fees, huge capital.

\section{Standards and Benefits of 4G}

This section outlines the details of 4G Standard and its various objectives, and comparison with existing 3G technologies. In telecommunications, $4 \mathrm{G}$ is the fourth generation of cellular wireless standards. It is a successor to the $3 G$ and $2 \mathrm{G}$ families of standards. $4 \mathrm{G}$ is described as MAGIC: mobile multimedia, any-time anywhere, global mobility support, integrated wireless solution, and customized personal service. In 2009, the ITU-R organization specified the IMT-Advanced (International Mobile Telecommunications Advanced) requirements for 4G standards, setting peak speed requirements for $4 \mathrm{G}$ service at $100 \mathrm{Mbit} / \mathrm{s}$ for high mobility communication (such as from trains and cars) and 1 Gbit/s for low mobility communication (such as pedestrians and stationary users). Initially, candidate technologies for 4G wireless standard are: (i) HSPA+ (High Speed Packet Access) (ii) UMB (Ultra Mobile Broadband) (iii) LTE (iv) Mobile WiMAX (v) XGP(eXtended Global Platform) [2].

A 4G system is expected to provide a comprehensive and secure all-IP based mobile broadband solution to laptop computer wireless modems, smart phones, and other mobile devices. Facilities such as ultra-broadband Internet access, IP telephony, gaming services, and streamed multimedia may be provided to users. 4G 
technologies such as mobile WiMAX, HSPA+, and firstrelease Long term evolution (LTE) have been on the market since 2006, 2008, and 2009 respectively. IMTAdvanced compliant versions of LTE and WiMAX are under development and called "LTE Advanced" and "Wireless MAN-Advanced" respectively. ITU has decided that LTE Advanced and Wireless MAN-Advanced should be accorded the official designation of IMT-Advanced. On December 6, 2010, ITU recognized that current versions of LTE, WiMax and other evolved 3G technologies that do not fulfill "IMT-Advanced" requirements could nevertheless be considered "4G", provided they represent forerunners to IMT-Advanced and "a substantial level of improvement in performance and capabilities with respect to the initial third generation systems now deployed. Basically 4G standard is based on two technologies: LTE Advanced standardized by the 3GPP and $802.16 \mathrm{~m}$ standardized by the IEEE (i.e. Wireless MAN-Advanced).

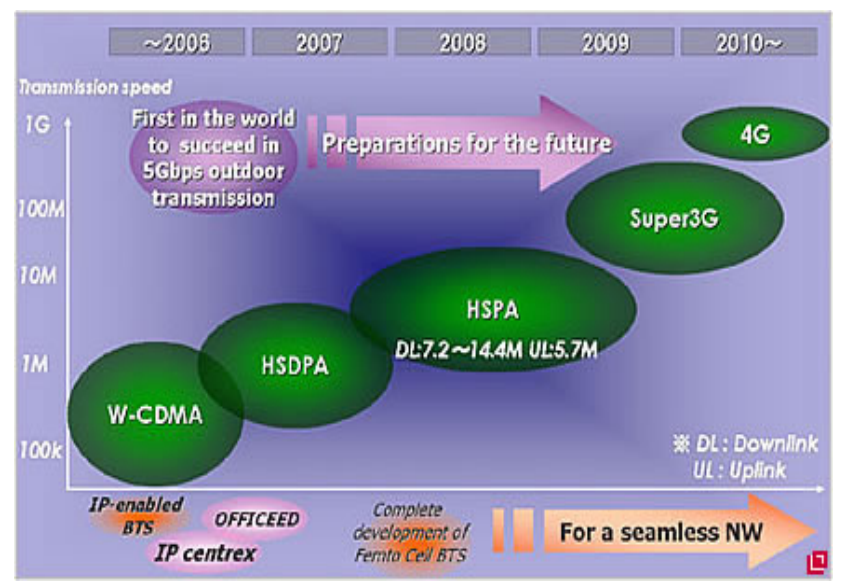

Figure 2. Evolution to 4G Technology

There are a number of advantages of $4 \mathrm{G}$ to answer one question "Why do we need 4G technologies?" 4G promises to deliver extremely high quality video of quality comparable to HD (high definition) TV. In addition $4 \mathrm{G}$ is being developed to accommodate the quality of service (QoS) and rate requirements set by further development of existing 3G applications like mobile broadband access, Multimedia Messaging Service (MMS), video chat, mobile TV, but also new services like HDTV. 4G may allow roaming with wireless local area networks, and may interact with digital video broadcasting systems.

Benefits of 4G includes: A data rate of at least 100 Mbit/s between any two points in the world. Smooth handoff across heterogeneous networks. Seamless connectivity and global roaming across a number of networks. High quality of service for next generation multimedia support (real time audio, high speed data, HDTV video content, mobile TV, etc.). Interconnection with existing wireless standards. An all IP, packet switched network. Also IP-based femtocells (home nodes connected to fixed Internet broadband infrastructure). 4G also has low cost since $4 \mathrm{G}$ systems can be built atop on existing networks and do not require operators to completely redesign and won't require carriers to purchase costly extra spectrum. In addition to being a lot more cost efficient, 4G is spectrally efficient, so carriers can do more with less. Table II below presents a brief difference between $4 \mathrm{G}$ and $3 \mathrm{G}$.
Table 2. 3G vs 4G

\begin{tabular}{|c|c|c|}
\hline & $3 G$ & $4 G$ \\
\hline $\begin{array}{c}\text { Major Requirement } \\
\text { Driving } \\
\text { Architecture }\end{array}$ & $\begin{array}{c}\text { Predominantly voice } \\
\text { driven-data was always } \\
\text { add on }\end{array}$ & $\begin{array}{c}\text { Converged data and } \\
\text { voice over IP }\end{array}$ \\
\hline $\begin{array}{c}\text { Network } \\
\text { architecture }\end{array}$ & Wide area cell based & $\begin{array}{l}\text { Hybrid -integration of } \\
\text { wireless LAN and } \\
\text { wide area }\end{array}$ \\
\hline Speed & $384 \mathrm{Kbps}$ to $2 \mathrm{Mbps}$ & $\begin{array}{l}20 \text { to } 100 \mathrm{Mbps} \text { in } \\
\text { mobile mode }\end{array}$ \\
\hline Frequency Band & $1800-2400 \mathrm{MHz}$ & $\begin{array}{l}\text { Higher frequency } \\
\text { bands (2-8 GHz) }\end{array}$ \\
\hline $\begin{array}{c}\text { Switching Design } \\
\text { Basis }\end{array}$ & Circuit and Packet & $\begin{array}{l}\text { All digital with } \\
\text { packetized voice }\end{array}$ \\
\hline $\begin{array}{c}\text { Access } \\
\text { Technologies }\end{array}$ & W-CDMA, & $\begin{array}{c}\text { OFDM and MC- } \\
\text { CDMA (Multi Carrier } \\
\text { CDMA) }\end{array}$ \\
\hline Component Design & $\begin{array}{l}\text { Optimized antenna } \\
\text { design, multi-band } \\
\text { adapters }\end{array}$ & $\begin{array}{l}\text { Smarter Antennas, } \\
\text { software multilane } \\
\text { and wideband radios }\end{array}$ \\
\hline IP & $\begin{array}{l}\text { A number of air link } \\
\text { protocols, including IP } \\
5.0\end{array}$ & All IP (IP6.0) \\
\hline
\end{tabular}

\section{Hardware and Software of 4G wireless}

This section presents the principal technological details of the $4 \mathrm{G}$ wireless system with respect to its hardware and software component of the physical layer.

\subsection{OFDM (Orthogonal Frequency Division Multiplexing)}

4G uses coded orthogonal frequency division multiplexing (COFDM) technology to combat the effects of Multipath fading and inter symbol interference (ISI). In addition Multi-carrier modulation (OFDM) in the downlink or single-carrier frequency-domain-equalization (SC-FDE) in the uplink is employed to exploit the frequency selective channel property without complex equalization. OFDM is derived from the fact that the high serial bit stream data is transmitted over large (parallel) number sub-carriers (obtained by dividing the available bandwidth), each of a different frequency and these carriers are orthogonal to each other.

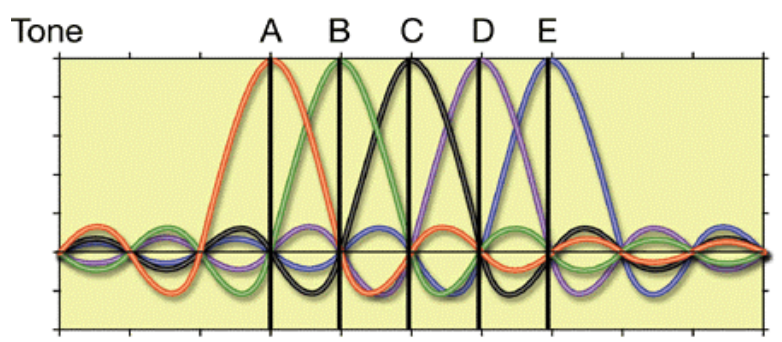

Figure 3. Orthogonal carriers in OFDM

OFDM converts frequency selective fading channel into $\mathrm{N}$ flat fading channels, where $\mathrm{N}$ is the number of subcarriers. Othogonality (as depicted in Figure 3) is maintained by keeping the carrier spacing multiple of $1 /$ Ts by using Fourier transform methods, where Ts is the symbol duration. Since channel coding is applied prior to OFDM symbol generation which accounts for the term 'coded' in COFDM. Turbo principle error-correcting codes is used to minimize the required SNR at the 
reception side. Figure 4 presents the simplified wireless OFDM block diagram.

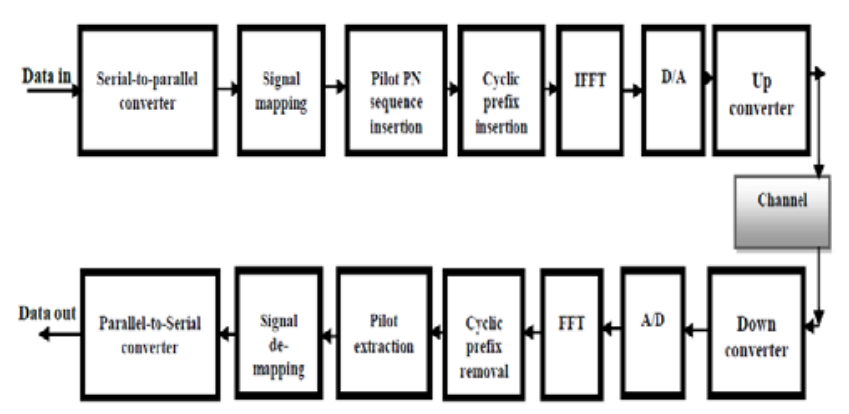

Figure 4. Wireless OFDM transceiver

\subsection{UWB (Ultra Wideband Networks)}

Ultra-Wideband (UWB) is a technology for transmitting message signal spread over a large bandwidth (>500 MHz) that should, in theory and under the right circumstances, be able to share radio spectrum with other users. One of the unique and valuable properties of UWB radio technology is the ability for a UWB radio system to determine "time of flight" of the direct path of the radio transmission between the transmitter and receiver at various frequencies. This helps to overcome multi path propagation, as at least some of the frequencies pass on radio line of sight. Another valuable aspect of pulse-based UWB is that the pulses are very short in space (less than $60 \mathrm{~cm}$ for a $500 \mathrm{MHz}$ wide pulse, less than $23 \mathrm{~cm}$ for a 1.3 $\mathrm{GHz}$ bandwidth pulse), so most signal reflections do not overlap the original pulse, and thus the traditional multipath fading of narrow band signals does not exist.

The secret to UWB is that it is typically detected as noise. This highly specific kind of noise does not cause interference with current radio frequency devices, but can be decoded by another device that recognizes UWB and can reassemble it back into a signal. Since the signal is disguised as noise, it can use any part of the frequency spectrum, which means that it can use frequencies that are currently in use by other radio frequency devices. UWB provides greater bandwidth - as much as 60 megabits per second, which is 6 times faster than today's wireless networks [3]. It also uses significantly less power, since it transmits pulses instead of a continuous signal. Figure 5 shows the UWB frequency of operation between 3.1 to 10.6 GHz [4].

\subsection{Advanced Antenna Systems}

The performance of $4 \mathrm{G}$ radio communications depends on an antenna system, termed smart or intelligent antenna.

Recently, multiple antenna technologies are emerging to achieve the goal of $4 \mathrm{G}$ systems such as high rate, high reliability, and long range communications. One technology, spatial multiplexing, gained importance for its bandwidth conservation and power efficiency. Spatial multiplexing involves deploying multiple antennas at the transmitter and at the receiver. Independent streams can then be transmitted simultaneously from all the antennas. This technology, called MIMO (as a branch of intelligent antenna), multiplies the base data rate by (the smaller of) the number of transmit antennas or the number of receive antennas. MIMO have been used to increase the system throughput and the reception reliability. Since UWB has almost impulse-like channel response, the combination with multiple antenna techniques is preferable as well. Coupling MIMO spatial multiplexing with UWB's already high throughput gives the possibility of short-range networks with multi-gigabit rates. Apart from this, the reliability in transmitting high speed data in the fading channel can be improved by using more antennas at the transmitter or at the receiver. This is called transmit or receive diversity. Both transmit/receive diversity and transmit spatial multiplexing are categorized into the space-time coding techniques, which does not necessarily require the channel knowledge at the transmitter.

There are two types of smart antennas: Switched Beam Antennas (as seen in Figure 6) have fixed beams of transmission, and can switch from one predefined beam to another when the User with the phone moves throughout the sector.

Adaptive Array Antennas (as seen in Figure 7) represent the most Advanced smart antenna approach to date using a variety of new signal processing algorithms to locate and track the user, minimize interference, and maximize intended signal reception [4]. Smart antennas can thereby: Optimize available power, Increase base station range and coverage, Reuse available spectrum, Increase bandwidth and Lengthen battery life of wireless devices.

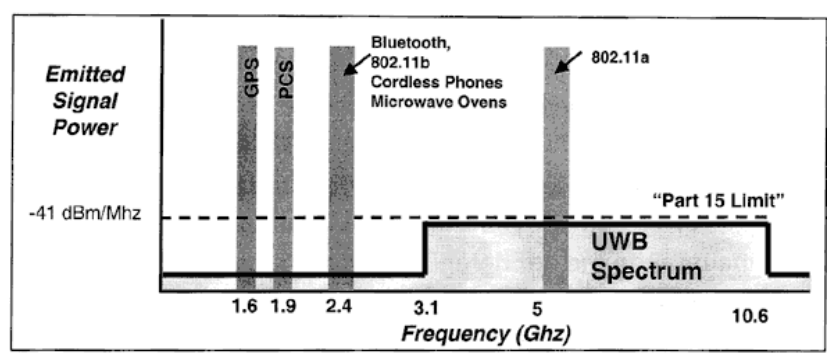

Figure 5. UWB frequency spectrum

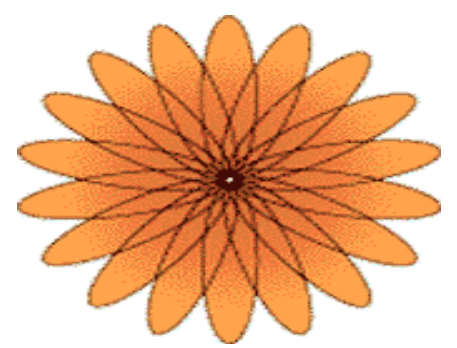

Figure 6. Switched Beam Antenna [4]

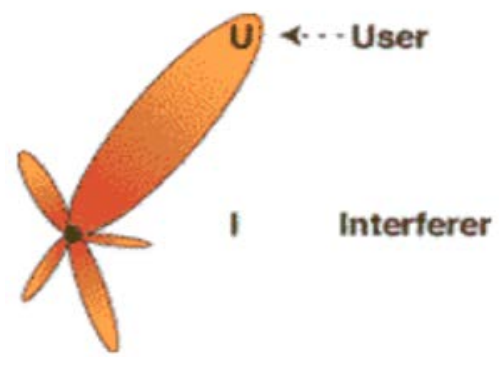

Figure 7. Adaptive Array Antennas [4]

\subsection{Adaptive Modulation and Power Control}


In a fading environment and for a highly loaded system there will almost exist users with good channel conditions. The modulation format for the scheduled user is selected according to the predicted signal to noise and interference ratio. By using sufficiently small time-frequency bins the channel can be made approximately constant within bins. For every timeslot, the time-frequency bins in the grid represent separate channels. For such channels the optimum rate and power allocation for maximizing the throughput can be calculated under a total average power constraint. The optimum strategy is to let one user, the one with best channel, transmit in each of the parallel channels. Figure 8 presents the adaptive modulation and coding concept.

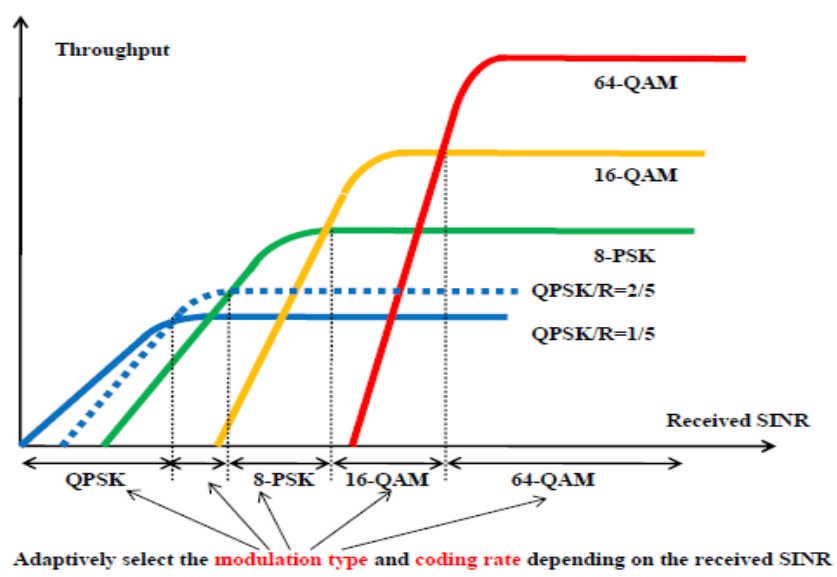

Figure 8. Adaptive modulation and coding

\subsection{Software Defined Radio (SDR)}

A software defined radio is one that can be configured to any radio or frequency standard through the use of software. A software defined radio in the context of 4G would be able to work on different broadband networks and would be able to transfer to another network seamlessly while traveling outside of the user's home network. A software defined radio's best advantage is its great flexibility to be programmed for emerging wireless standards. It can be dynamically updated with new software without any changes in hardware and infrastructure. Software could just download interface automatically. This is the job of the packet layer, which will split the data into small "packets.” A Conceptual definition of the software defined radio (SDR) is presented in Figure 9.

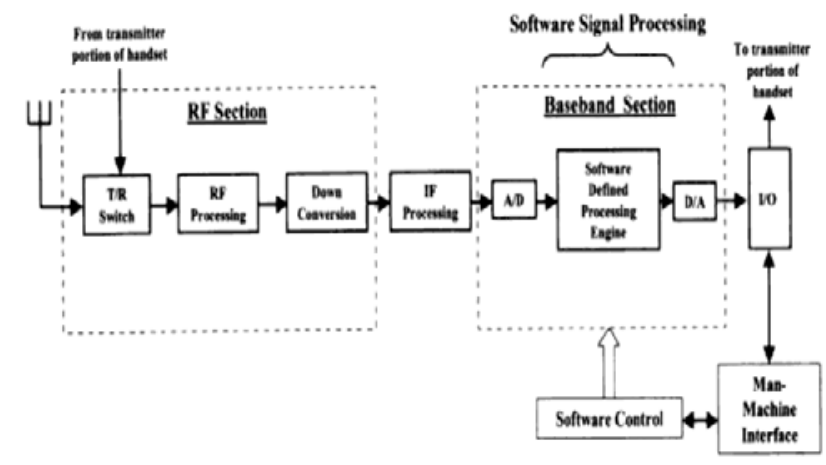

Figure 9. Conceptual definition of the software defined radio (SDR) [9]

\subsection{Packet Layer IPv6}

The next generation 4G addressing system uses the Internet Protocol version 6 (IPv6) to locate devices. IPv6 has a much larger address space. Its addresses take the form $\mathrm{x}$ : $\mathrm{x}$ : $\mathrm{x}$ : $\mathrm{x}$ : $\mathrm{x}$ : $\mathrm{x}$ : $\mathrm{x}$ : $\mathrm{x}$ where each $\mathrm{x}$ is the hexadecimal value that makes up one eighth of the address. An example of this is: FEDC: BA98: 7654: 3210: FEDC: BA98: 7654: 3210 (The Internet Engineering Task Force Network Working Group). Using this address format, there is room for approximately $3.40 * 1038$ unique addresses. This is approximately $8.05 * 1028$ times as large as the IPv4 address space and should have room for all wired and wireless devices, as well as room for all of the foreseeable expansion in several lifetimes. There are enough addresses for every phone to have a unique address. Thus, phone in the future can use VoIP over the Internet instead of continuing to use their existing network.

\section{Barriers in Deployment of 4G}

The security issues of 4G wireless networks and systems are a challenging one. Principal issues encountered by designers engineers of 4G wireless security include the following: (i) One of the common Security issues for $4 \mathrm{G}$ mobile wireless devices includes when it comes to accessing of the Internet from a fixed location with the additional requirements for flexibility and mobility (ii) Whenever additional cryptographic methods and security mechanisms will be applied to IP networks, there will be an obvious impact on the performance and traffic handling capacity of the service provider's network (iii) The Physical Layer Issues are related to both WiMAX and LTE in the sense that both are prone to Interference and Scrambling attacks but these can be minimized to some extent [2]. (iv) Integrating the IPbased systems with existing non-IP systems and thereby providing QoS (Quality of Service) guarantee for end-toend services that involve different systems is also a big problem (v) Further Charging/ billing is a big challenge since to collect, manage and store the customers' accounts information from various service providers is difficult task [1].

\section{Future Scope of $4 \mathrm{G}$}

There are many good reasons for $4 \mathrm{G}$ development and a variety of current and evolving technologies to make $4 \mathrm{G}$ a reality. Both service providers and users want to reduce the cost of wireless systems and the cost of wireless services. Lesser the expensive of the system, the more people who will want to own it. 4G's flexibility will allow the integration of several different LAN and WAN technologies. Finally, the $4 \mathrm{G}$ wireless system would truly go into a "one size fits all" category, having a feature set that meets the needs of just about everyone.

\section{Conclusion}

This paper briefly described the 4G wireless technology as next generation networks. It presented the 4G architecture, the technologies involved, 4G standard, benefits, challenges in deployment, design for the LTE 
and WiMAX technologies and security issues. It can be finally concluded that the ever increasing demands of the customers can be only fulfilled by one and only one the 4G wireless system. Today's wired society is going wireless and if it has problem, 4G is answer. 4G will be an intelligent technology that will be fast replacing 3G. But the final success of $4 \mathrm{G}$ mobile communication systems will depend upon when it is fully implemented and the new services and contents made available to users.

\section{References}

[1] Afaq H. Khan, Mohammad A Qadeer, Juned A Ansari, and Saria Waheed, "4G as a Next Generation Wireless Network," International Conference On Future Computer And Communication, pp. 334-338, March, 2009.
[2] N. Seddigh, B. Nandy, R. Makkar and J.F. Beaumont, "Security Advances and Challenges in 4G Wireless Networks," 2010 Eighth Annual International Conference on Privacy, Security and Trust, pp. 62-71, June, 2010.

[3] Jawwad Ahmad, Ben Garrison, Jim Gruen, Chris Kelly, and Hunter Pankey, "4G Wireless Systems," Next-Generation Wireless Working Group, May, 2003.

[4] Manu. R, "4G Wireless Systems," Technical Seminar report, Kalpataru Institute of Technology, Belgaum, Karnataka, 20092010.

[5] H. Harada \& Ramjee Prasad, Simulation and Software Radio for mobile communications: Artech House, 2003.

[6] John. G. Proakis, "Digital Communications", 3rd edition, McGraw-Hill, 1995.

[7] Walter Tuttlebee, “Software Defined Radio”, 2002 John Wiley \& Sons, Ltd.

[8] "UMTS LTE Network Architecture", Technical specification TS 23.002, version 8.4.0 (Release 8), 3GPP.

[9] ITU-R M. 1645: Framework and overall objectives of the future development of IMT-2000 and systems beyond IMT-2000.

[10] http://en.wikipedia.org/wiki/4G. 